\title{
The narrow leaf mutant (nlf/nlf) of maize (Zea mays L.)
}

\author{
Victoria C. Cheng, David B. Walden and Ping-chin Cheng \\ *Williamsville East HS, Williamsville, NY \\ **Department of Biology, University of Western Ontario, London, Ontario, Canada \\ ***Advanced Microscopy and Imaging Lab., Dept of Electrical Eng., SUNY at Buffalo, NY 14260
}

A narrow leaf mutant (nlf/nlf) of maize (Zea mays L.) was identified by one of the authors (DBW) few years ago (FIG 1). Seed of this mutant may be obtained currently from D. B. Walden or from the Maize Genetic Coop in 2007. The purpose of this study is to document the phenotype in detail. nlf/nlf plants were grown to maturity in the nursery at the University of Western Ontario, London, Ontario, Canada during the summers of 2004 and 2005. Leaf width (mid-blade) and length were measured. Five leaves were measured (two below and two above the main ear insertion node; designated as $-2,-1,0,+1,+2$ respectively). The phenotype 1 to 5 shown in FIG 2 represents progenies from different selfings. No significant difference between the 5 groups. The five columns in each phenotype are data from leaves $-2,-1,0,+1,+2$ respectively (from left to right) The leaf blade length of $n l f / n l f$ shows no significant difference from wild type $(+/+)$ while the width of the leaf blade exhibits a $40 \%$ reduction (FIG 2).

The size of leaf epidermal cells of $n l f / n l f$ were studied by using a surface replica technique obtained from growth-chamber grown material [1] and as described by Cheng et al. [2]. In summary, the surface replica were obtained by applying a thin layer of clear nail enamel (New York Color ${ }^{\mathrm{TM}} 138 \mathrm{~B}$ consists of isopropyl alcohol, butyl acetate, ethyl acetate, nitrocellulose, tosylamide/formaldehyde resin, n-butyl alcohol, dibutyl phphalate, camphor, acrylate copolymer, and benzophenone-1) coating on the surface of leaf blades. After allowing the nail enamel to dry, the coating was carefully peeled off from the surface of the specimen and floated on a drop of water on a glass slide. The slide was then heated slightly to flatten the replica and then the water was carefully withdrawn [2]. It is important to float the replica with its cell-replicating surface facing away from the microscope slide. Optical microscopy was performed on an Olympus BX51 upright microscope equipped with an Edge dynamic oblique illumination condenser [3] [4] and a Nikon D100 digital camera. The length and width of epidermal cells (only epidermal cells in files containing no stomata were measured) were measured from micrographs (adaxial surface, mid-blade of \#0 leaf) (FIG 3 and 4). The data indicates no significant reduction in both cell width and length in $n l f / n l f$ leaf (FIG 5). Our result suggest that the $n l f$ mutation reduces the width of leaves (nearly by $1 / 2$ ) but not length by reducing the number of cells/files rather than by altering (at least for epidermal cells) the individual cell dimensions Additional studies on cell number, file number, and cell types will be conducted in the 2006 summer nursery.

[1] V. C. Cheng et al., Microsc. Microanal., 8 (2002) 1038CD)

[2] P. C. Cheng et al., Microsc. Microanal. 7 (Suppl. 2) (2001) 100

[3] G. Greenberg and A. Boyde, In: Focus on Multidimensional Microscopy, Vol. 1, Eds. PC Cheng et al., World Scientific Publishing, (1999) 1-24;

[4] A. Boyde et al., Scanning 23 (2001), 84

The authors are grateful to Dr. G. Greenburg for providing the dynamic condenser for this study. 

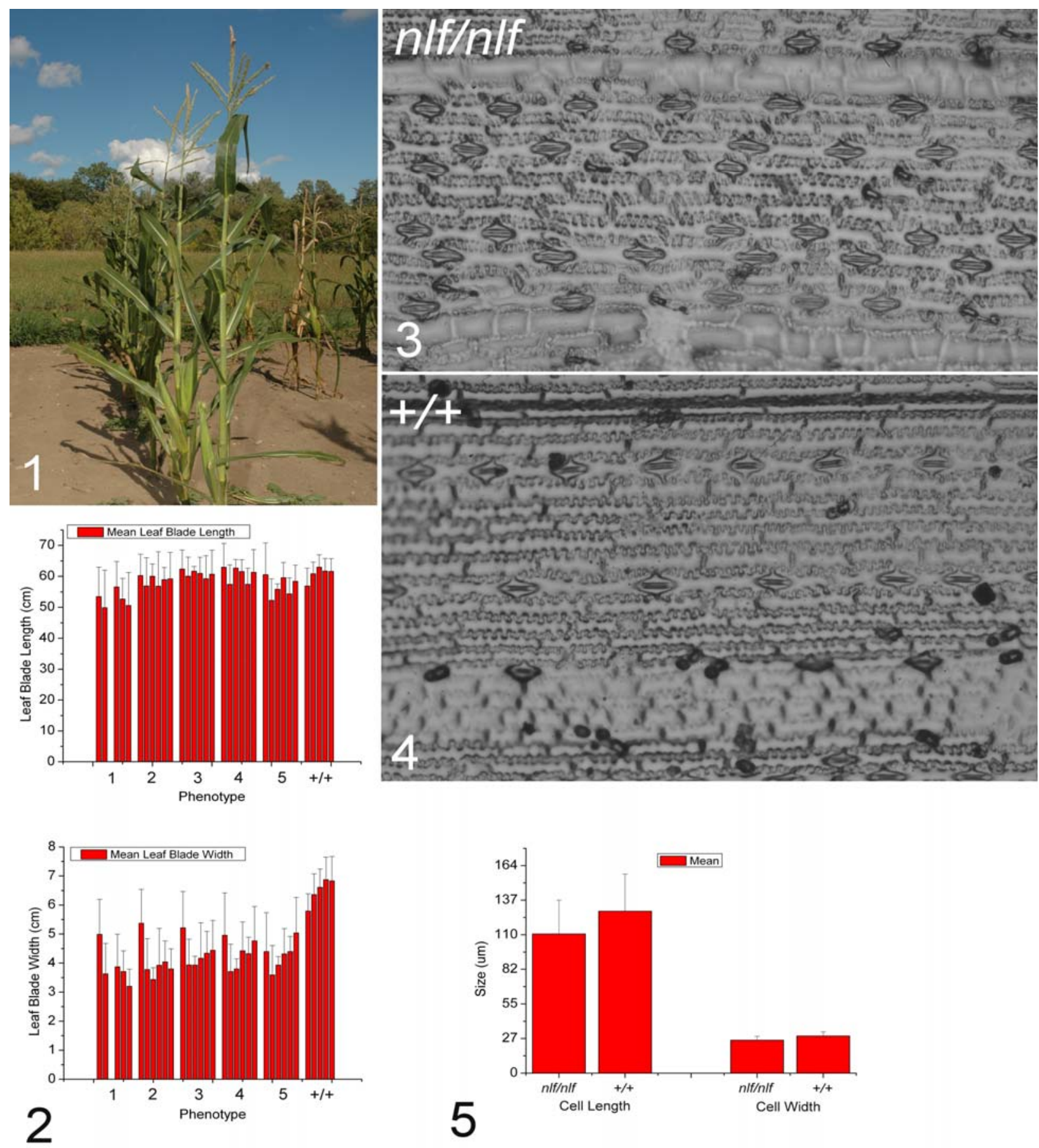

FIG 1. nlf/nlf plant (2005 Summer nursery, London, Ontario, Canada).

FIG 2. The leaf length and width of $n l f / n l f$ and wild type $(+/+)$. The five columns in each group represent data from $-2,-1,0$ (main ear insertion node), +1 and +2 leaves respectively (left to right). Missing column in group 1 is due to lost of leaves due to environmental causes. (Vertical bar: standard deviation)

FIG 3 and 4. Adaxial surface replica obtained from the mid-blade of \#0 leaf (main ear insertion node) of $n l f / n l f$ and $+/+$ respectively..

FIG 5. Comparison of the width and length of epidermal cells between $n l f / n l f$ and $+/+.(n=140)$ 
https://doi.org/10.1017/S143192760606569X Published online by Cambridge University Press 